\title{
miR-520c-3p regulates IL-1 $\beta$-stimulated human chondrocyte apoptosis and cartilage degradation by targeting GAS2
}

\author{
Le Peng ${ }^{\dagger}$, Ming Deng ${ }^{\dagger}$, Yonggang Ma, Wei Hu and Fan Liang
}

\begin{abstract}
Background: MicroRNAs (miRNAs) have been shown to be associated with osteoarthritis $(\mathrm{OA})$ progression. This study aimed to explore the role of miR-520c-3p in OA progression.

Methods: Expression levels of miR-520c-3p and Growth arrest-specific 2 (GAS2) were detected using quantitative real-time PCR. The proliferation and apoptosis of cells were measured using cell counting kit 8 (CCK8) assay and flow cytometry. Furthermore, the protein levels of apoptosis-related markers, extracellular degradation markers, inflammatory response markers, and GAS2 were tested using quantitative real-time polymerase chain reaction (RTPCR) and western blot (WB) analysis. In addition, the interaction between miR-520c-3p and GAS2 was examined using dual luciferase reporter assay.

Results: GAS2 was highly expressed, and miR-520c-3p was lowly expressed in OA cartilage tissues. miR-520c-3p could promote the proliferation and inhibit the apoptosis and inflammation of OA chondrocytes. miR-520c-3p could be sponged by GAS2, and its inhibitor could reverse the regulation of GAS2 on the biological functions of OA chondrocytes. GAS2 was a target of miR-520c-3p, which was identified by bioinformatic analysis and dualluciferase reporter assay. Overexpression of GAS2 could inhibit the proliferation and promoted the apoptosis and inflammation of OA chondrocytes.
\end{abstract}

Conclusion: Our data showed that miR-520c-3p might regulate the GAS2 to inhibit the progression of OA.

Keywords: miR-520c-3p, GAS2, Osteoarthritis, Apoptosis

\section{Background}

Osteoarthritis (OA) is degenerative disease and is a major cause of pain and physical disability [1]. Approximately $15 \%$ of the global population suffer from OA [2]. Genetics, aging, obesity, and mechanical stress are the primary causes of OA [3]. Clinical therapy for OA includes nonsteroidal anti-inflammatory drugs (NSAIDs), platelet-rich plasma (PRP), or hyaluronic acid (HA) injections $[4,5]$. However, currently no ideal treatment

\footnotetext{
* Correspondence: li99770@163.com

${ }^{\dagger}$ Le Peng and Ming Deng contributed equally to this article and should be listed as co-first authors.

Department of Orthopaedics, Renmin Hospital of Wuhan University, No. 238, Jiefang Road, Wuchang District, Wuhan City 430060, Hubei Province, China
}

strategies have been developed to prevent the OA progression [6]. Chondrocytes are responsible for normal maintenance and remodeling of articular cartilage and extracellular matrix [7]. The inflammatory mediator interleukin 1 beta (IL-1 $\beta$ ) or tumor necrosis factor- $\alpha$ (TNF- $\alpha$ ) promotes inflammation response, extracellular matrix degradation, and chondrocyte apoptosis and finally causes cartilage damage [8].

Although there are numerous factors involved in the development of OA, IL- $1 \beta$ has been reported to play a dominant role in the pathogenesis of OA. IL-1 $\beta$ can significantly increase cartilage damage and thus used to mimic OA in vitro studies [9]. Therefore, fully 
elucidated, the molecular mechanisms of OA are imperative for the development of novel therapeutic strategies.

MicroRNAs (miRNAs), small noncoding RNAs that function in the posttranscriptional regulation of genes, are involved in a number of physiological functions and disease processes, including OA [10]. In previous studies, it was found that the occurrence and development of $\mathrm{OA}$ are accompanied by changes in various miRNAs. Zhang et al. [11] found that miR-132 regulates the development of OA through modulation of PTEN/PI3K/AKT signaling pathway. Zhang et al. [12] revealed that miR$130 \mathrm{~b}$ downregulation potentiates chondrogenic differentiation of bone marrow mesenchymal stem cells by targeting SOX9. However, the pathogenesis of OA is still far from being fully understood. miR-520c-3p has been found to mediate osteosarcoma progression [13], vascular endothelium dysfunction [14], and cholangiocarcinoma progression [15]. We conducted a bioinformatic analysis and found that miR-520c-3p was upregulated in OA-affected tissue than normal cartilage tissue. Accordingly, it may be speculated that miR-520c-3p plays an important role in the initiation and progression of OA.

Mechanism of action of miRNA was that miRNA can affect target gene expression through a 3'-untranslated region binding manner. Growth arrest-specific 2 (GAS2) gene was originally identified in a genetic screen of murine fibroblasts that were cultured under growth arrest conditions [16]. Recently, researcher found that GAS2 was involved in the regulation of apoptosis and chondrogenesis in the developing mouse limb [17]. Through bioinformatic analysis, we found that miR-520c-3p has putative binding sites with GAS2.

In this study, we found that the overexpression of GAS2 was able to reverse the protective effect of miR$520 c-3 p$ on IL-1 $\beta$-induced chondrocyte apoptosis. These findings may substantiate miR-520c-3p as a new therapeutic target for the treatment of OA.

\section{Methods}

\section{Clinical samples and cell culture}

Cartilage tissue samples were collected from patients $(n=20)$ with end-stage symptomatic hip OA and patients $(n=20)$ without OA histories by total hip arthroplasty at Renmin Hospital of Wuhan University in this study, which was endowed by the Ethics Committee of Renmin Hospital of Wuhan University. And written informed consent was signed by all tissue donors involved in the present research.

Under a humidified incubator with $5 \% \mathrm{CO}_{2}$ at $37^{\circ} \mathrm{C}$, human chondrocyte (American Type Culture Collection, Manassas, VA, USA) were allowed to grow in Dulbecco's modified Eagle's medium (DMEM; Gibco, Carlsbad, CA, USA) with $10 \%$ fetal bovine serum (FBS; Gibco) and 0.1
mg/mL G-418 (Sigma-Aldrich, St. Louis, MO, USA). For the establishment of the cell model of OA, chondrocytes were induced with $0,5,10$, and $15 \mathrm{ng} / \mathrm{mL}$ of IL- $1 \beta$ (Sigma-Aldrich) for multiple time points $(0 \mathrm{~h}, 6 \mathrm{~h}, 12 \mathrm{~h}$, $24 \mathrm{~h}$, and $48 \mathrm{~h}$ ). Besides, $10 \mathrm{ng} / \mathrm{mL}$ of IL- $1 \beta$ treatment for $24 \mathrm{~h}$ was selected for further functional experiments.

\section{Real-time quantitative polymerase chain reaction (RT- qPCR)}

According to the producer's instructions of TRIzol reagent (Invitrogen, Carlsbad, CA, USA), total RNAs from tissues and cells were extracted, followed by reverse transcription using PrimeScript RT Reagent Kit (TaKaRa, Tokyo, Japan). On Thermal Cycler CFX6 System (Bio-Rad, Hercules, CA, USA), RT-qPCR reaction was performed referring to the operation manual SYBR Green PCR Kit (TaKaRa). After normalization with GAPDH for Cas-3, Bcl-2, MMP-13, COL2A1, IL-6 and IL-8 and U6 for miR-520c-3p, the levels of gene were calculated by the $2^{-\Delta \Delta \mathrm{Ct}}$ method. The specific primers were displayed as: miR-520c-3p: 5'-GGAAGTGGTG GCTATGAGTCAG-3' (sense), 5'-TGTCAATTTG AAACTTAAAAAGCAG-3' (antisense); Cas-3: 5' CTGCCACAGAACCAGTTCCC-3' (sense), 5' CTGTGACACGCCTGTTTGGG-3' (antisense); Bcl-2: 5'-GCATCGTTCCTTCAAGCCGATCT-3' (sense), 5' TGGGTGAGTCGTTCGG-3' (antisense); MMP-13: 5' ATACGTGGATTGAGGACCACT-3' (sense), 5' '-TCCA ATGTCAAGTAGCGGTTG-3' (antisense); COL2A1: 5'-CTCGCTTCGGCAGCACA-3' (sense), 5' -CTGCCT TCCTGCACCAAGTA-3' (antisense); IL-6: 5' AUTTCGCAGTTGCAT-3' (sense), 5' -TGGAACAGCA CGGATTTGGA-3' (antisense); IL-8: 5'-GGCAATGC CATTCTACAGATACT-3' (sense), R 5'-CAACCGAG AAATCCAGCACCT-3' (antisense); GAS2: F 5'-TGGA ACAGCACGGATTTGGA-3' (sense), R 5'-CTGCCTTC CTGCACCAAGTA-3' (antisense); GAPDH: 5'-GTCA ACGGATTTGGTCTGTATT-3' (sense), 5'-AGTCTT CTGGGTGGCAGTGAT-3' (antisense).

\section{Cell viability assay}

After treatment with IL-1 $1 \beta$, transfected and untransfected chondrocytes $\left(5 \times 10^{3}\right.$ cells/well $)$ were introduced into 96-well plates, followed by incubation for 24 h. Whereafter, $10 \mu \mathrm{L}$ CCK-8 (Dojindo, Kumamoto, Japan) was added into each well for another $4 \mathrm{~h}$. Based on the user's guidebook of a microplate reader (Bio-Rad, Hercules, CA, USA), the absorbance at $450 \mathrm{~nm}$ was determined.

\section{Western blot assay}

For total protein preparation, chondrocytes were treated with pre-cold RIPA buffer (Beyotime, Nantong, China), followed by quantification using bicinchoninic acid 
(BCA) Protein Assay Kit (Solarbio, Beijing, China). For immunoblotting, the protein samples $(50 \mu \mathrm{g})$ were separated by $10 \%$ SDS-PAGE and electrophoretically transfected to nitrocellulose membranes (Millipore, New York, NY, USA). And then, the membranes were subjected to hybridize with primary antibodies: cleaved cas3 (1:1000, Abcam, Cambridge, MA, USA), Bcl-2 (1:1000, Abcam, Cambridge, MA, USA), MMP-13 (1:1000, Abcam, Cambridge, MA, USA), COL2A1 (1:1000, Abcam, Cambridge, MA, USA), IL-6 (1:1000, Abcam, Cambridge, MA, USA), IL-8 (1:1000, Abcam, Cambridge, MA, USA), and GAPDH (1:1000, Abcam, Cambridge, MA, USA) at $4^{\circ} \mathrm{C}$ overnight. After incubation with secondary antibody (1:10,000, Abcam, Cambridge, MA, USA) for $2 \mathrm{~h}$, the detection of protein bands was performed using ECL detection system (GE Healthcare, Piscataway, NJ, USA).

\section{Cell transfection}

For miR-520c-3p overexpression, chondrocytes were transfected with $50 \mathrm{nM}$ of miR-520c-3p overexpression plasmid (miR-520c-3p, RiboBio, Guangzhou, China) and its negative control NC mimic (RiboBio). For GAS2 overexpression, the sequence of GAS2 was introduced into pcDNA empty vector (vector, Invitrogen, a negative control) to obtain pcDNA-GAS2 (GAS2) vector, and then, these vectors $(200 \mathrm{ng})$ were transfected into chondrocytes. In this study, all transfection was employed according to the instructions Lipofectamine 3000 (Invitrogen) for $48 \mathrm{~h}$.

\section{Cell apoptosis assay}

Generally, treated chondrocytes were collected and washed, followed by re-suspending Binding Buffer. After incubation with $5 \mu \mathrm{L}$ Annexin (V-fluorescein isothiocyanate) V-FITC and $10 \mu \mathrm{L}$ propidium iodide (PI) (Roche, Indianapolis, IN, USA) for $15 \mathrm{~min}$, the measurement of the apoptosis rate was conducted following the operation manual of a FACS Calibur (BD Bioscience, San Jose, CA, USA).

\section{Dual-luciferase reporter assay}

In this assay, the binding relationship between miR-520c$3 p$ and GAS2 was predicted by TargetScan software, as verified by a dual-luciferase reporter assay. In short, the GAS2 wild-type (WT) reporter vector (WT-GAS2) 3' untranslated region ( $3^{\prime}$ UTR) were generated by inserting the fragment sequence of GAS2 3'UTR with the putative binding site of miR-520c-3p into pmirGLO plasmids (Promega, Madison, WI, USA). Analogously, the corresponding mutant fragments of GAS2 3'UTR were applied to build the vectors Mutant (MUT)-GAS2 3'UTR. Whereafter, the constructed vectors were transfected into chondrocytes along with miR-520c-3p or NC mimic, in line with the user's guidebook of Lipofectamine 3000 reagent (Invitrogen). At length, the assessment of the luciferase activities was carried out using a dual-luciferase reporter assay kit (Promega) after transfection for $48 \mathrm{~h}$.

\section{Statistical analysis}

SPSS statistical software (version 20.0; IBM SPSS, Armonk, NY, USA) was used to conduct the statistical analysis. All data were presented as mean \pm standard deviation from 3 independent experiments. Results from three or more groups were analyzed by one-way analysis of variance followed by Tukey's multiple comparison test. Statistical analysis was performed by Student's $t$-test when two groups were analyzed. $P<0.05$ was considered statistically significant.

\section{Results}

MiR-520c-3p was upregulated in OA-affected cartilage and inhibited the IL-1 $\beta$-induced chondrocytes' apoptosis

In order to identify the function of miR-520c-3p for the chondrocytes, we constructed miR-520c-3p mimic and the transfection efficacy was identified by RT-PCR. After transfected with miR-520c-3p mimic, the miR-520c-3p expression increased about 8 -fold than NC mimic (Fig. 1a). Then, CCK- 8 assay was performed to identify the role of miR-520c-3p for cell viability. Results suggested that IL-1 $\beta$ significantly decreased the cell viability at 12 , 24, 36, and $48 \mathrm{~h}$ (Fig. 1b). The partial inhibition of cell viability by IL- $1 \beta$ could be reversed by addition of miR$520 \mathrm{c}-3 \mathrm{p}$ at 36 and $48 \mathrm{~h}$ (Fig. 1b). To identify the relative expression of miR-520c-3p between normal and OAaffected cartilage, RT-PCR was performed. We found that miR-520c-3p was significantly downregulated in OA-affected cartilage (Fig. 1c, $P<0.05$ ). Moreover, the relative expression of miR-520c-3p was significantly downregulated after treatment with IL-1 $\beta(10 \mathrm{ng} / \mathrm{ml})$ at 12, 24, 36, and $48 \mathrm{~h}$ (Fig. 1d, $P<0.05$ ). Then, different concentration of IL-1 $\beta$ were treated with chondrocytes; we found that a significantly downregulated miR-520c$3 p$ was observed after IL- $1 \beta$ concentration increased (Fig. 1e, $P<0.05$ ). However, the expression did not change significantly when the IL- $1 \beta$ concentration was further increased to $15 \mathrm{ng} / \mathrm{ml}$ (Fig. 1e, $P>0.05$ ). In addition, cell apoptosis rates were detected using flow cytometry. As shown in Fig. 1f, IL-1 $\beta$ resulted in significant increase in apoptosis incidence $(228 \%)$ than control group. While miR-520c-3p mimic significantly inhibited IL-1 $\beta$-induced apoptosis (Fig. 1f).

\section{MiR-520c-3p significantly downregulated apoptotic-} related, inflammation response markers and upregulated extracellular matrix-associated markers

To further identify the role of miR-520c-3p on the apoptosis, extracellular degradation and inflammation 


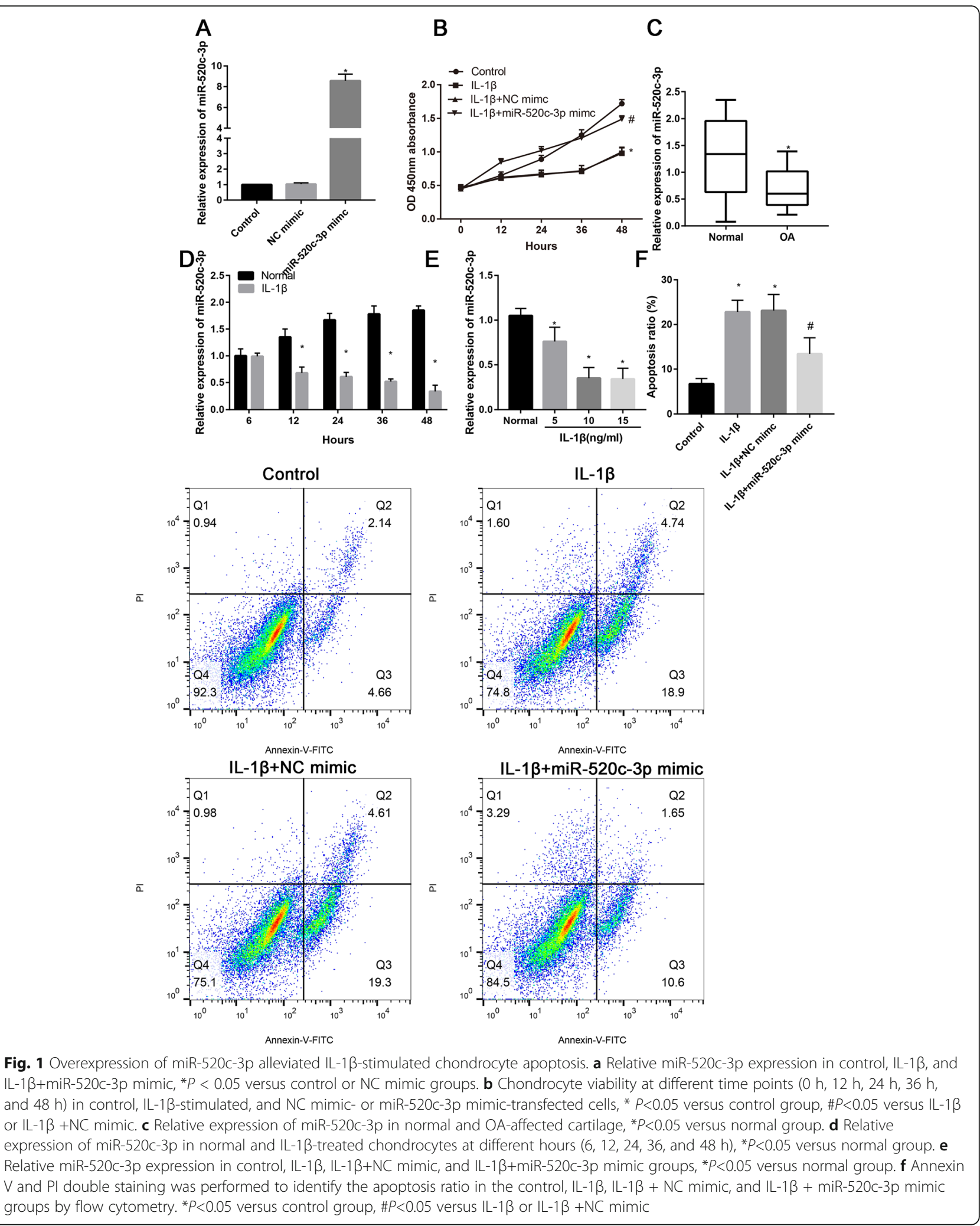

response. RT-PCR and western blot were performed to identify the apoptotic-related, inflammation response markers and extracellular matrix-associated markers.
We found that IL-1 $\beta$ significantly increased the caspase3 expression, while decreased the $\mathrm{Bcl}-2$ expression. After co-transfected with miR-520c-3p mimic, this trend is 
A

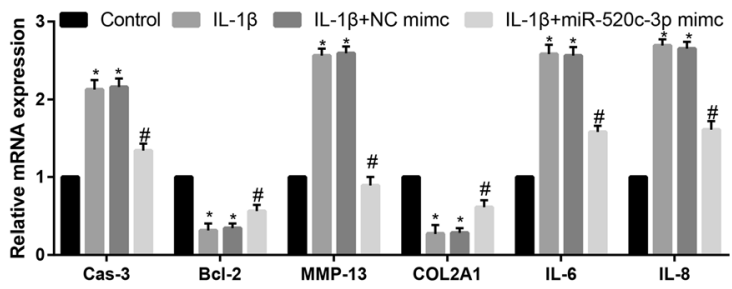

\section{B}
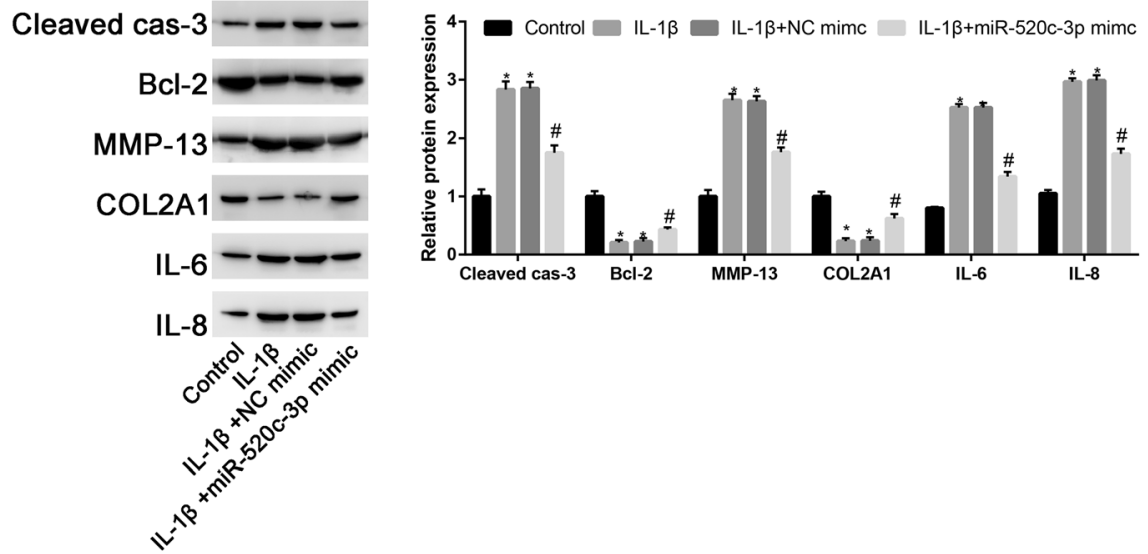

Fig. 2 Protective role miR-520c-3p in alleviating $I L-1 \beta$ induced the apoptosis, inflammation response, and matrix degradation of chondrocytes. a qRT-PCR to assess the mRNA levels of caspase-3, BCl-2, MMP-13, COL2A1, IL-6, and IL-8 in control, IL-1 $\beta, I L-1 \beta+N C$ mimic, and IL-1 $\beta+$ miR-520c$3 p$ mimic groups. $\mathbf{b}$ Western blot assay to evaluate the protein levels of cleaved-caspase-3, BCl-2, MMP-13, COL2A1, IL-6, and IL-8 in control, IL-1 $\beta$, $\mathrm{IL}-1 \beta+\mathrm{NC}$ mimic, and $\mathrm{IL}-1 \beta+$ miR-520c-3p mimic groups

reversed (Fig. 2a). Then, we measured the expression of MMP-13, which is a biochemical marker of collagenase for cartilage degeneration. Results revealed that IL-1 $\beta$ significantly increased the MMP-13 expression; this trend is reversed by miR-520c-3p mimic (Fig. 2a). Moreover, IL- $1 \beta$ significantly decreased the COL2A1 expression, which is a characteristic chondrogenic genes. And the inhibition effects of IL-1 $\beta$ on COL2A1 expression was partially reversed by miR-520c-3p mimic (Fig. 2a). Then, we assessed the miR-520c-3p on the markers of inflammation (IL-6 and IL-8). IL-1 $\beta$ significantly increased the IL- 6 and IL-8 expression. However, these effects were partially reversed by miR-520c-3p mimic. RTPCR results validation with Western Blot (Fig. 2b).

\section{MiR-520c-3p inhibited the IL-1 $\beta$-induced chondrocytes' apoptosis through directly target with GAS2}

To determine the target gene of miR-520c-3p, we performed a Venn diagram analysis to predict its downstream target gene from Targetscan, miRanda, and miRDB databases. Results are shown in Fig. 3. We found 20 genes that overlap in Targetscan, miRanda, and miRDB databases.

Overexpressing miR-520c-3p inhibited the luciferase activity of GAS2-wt and failed to alter GAS2-mut activity (Fig. 4a). And the GAS2 was upregulated in OAaffected cartilage than normal cartilage (Fig. 4b). Moreover, the relative expression of GAS2 was significantly upregulated after treatment with IL-1 $\beta(10 \mathrm{ng} / \mathrm{ml})$ at 12 , 24, 36, and $48 \mathrm{~h}$ (Fig. 4c, $P<0.05$ ). It was observed that GAS2 expression increased with the increase in concentrations of IL- $1 \beta$. When the IL-1 $\beta$ concentration increased to $15 \mathrm{ng} / \mathrm{ml}$, the GAS2 expression did not change too much (Fig. $4 \mathrm{~d}, P>0.05$ ). There was a significant negative correlation between miR-520c-3p expression and GAS2 expression (Fig. $4 \mathrm{e}, P<0.05$ ). Consistent with previous findings, IL- $1 \beta$ significantly increased GAS2 expression, while the upregulation effects were partially reversed by miR-520c-3p mimic (Fig. 4f, $P<$ $0.05)$. Western blot analysis was in agreement with the RT-PCR results, showing that the IL- $1 \beta$ increased GAS2 expression and miR-520c-3p mimic partially reversed the promotion effects of miR-520c-3p mimic on GAS2 expression.

\section{GAS2 could partially reversed the miR-520c-3p on the apoptosis of chondrocytes}

To further explore the mechanism of miR-520c-3p on the biological behavior of chondrocytes. GAS2 


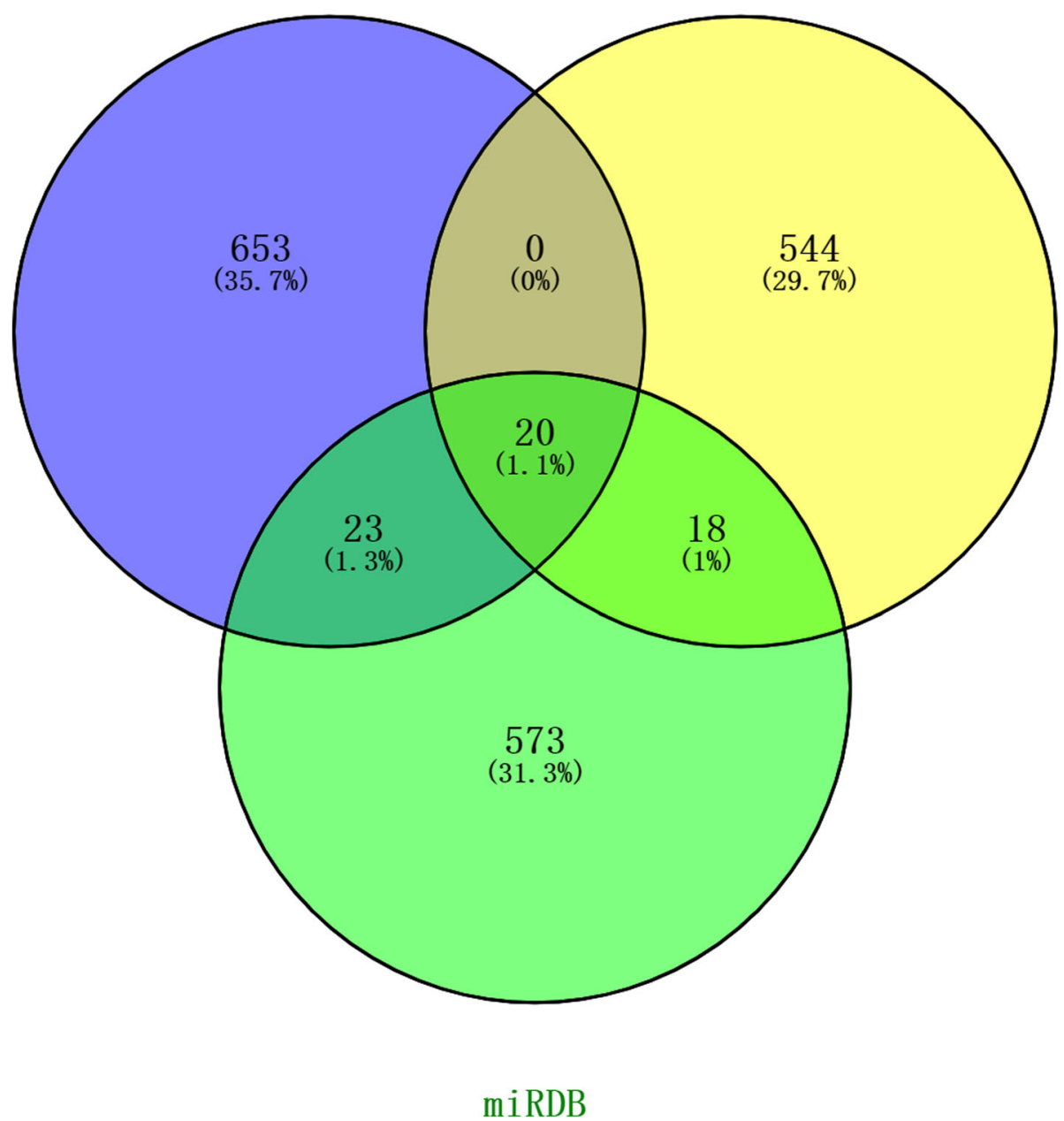

Fig. 3 Venn diagram of the miR-520c-3p target genes from Targetscan, miRanda, and miRDB databases

overexpression plasmid was constructed. As illustrated in Fig. 5a, the promotion effects of miR-520c-3p on cell viability was partially blocked by GAS overexpression plasmid. Meanwhile, the inhibition effects of miR-520c-3p on the apoptosis of chondrocytes were partially reversed by GAS2 overexpression (Fig. 5b). To illustrate the potential mechanism of GAS2 on the apoptosis of chondrocytes. RT-CPR was performed to identify the miR-520c-3p for apoptotic, matrix degradation and inflammation response change. Results suggested that GAS2 overexpression could partially reverse the miR-520c$3 p$ on the caspase-3, Bcl-2, MMP-13, COL2A1, IL-6, and IL8 expression (Fig. $5 \mathrm{c}$ ). Western Blot analyses were conducted to confirm the results of RT-CPR (Fig. 5d).

\section{Discussion}

The occurrence of $\mathrm{OA}$ is a complex process involving multiple factors, and its etiology and pathogenesis are still not very clear. A great amount of evidence shows that the degeneration of articular cartilage is considered to be the most important pathological link that causes OA. Chondrocytes, as the only cellular component of cartilage, their biological characteristics are closely related to the development of OA.

Here, we explored the role of a new miRNA, miR$520 c-3 p$ in the progression of OA by assessing its function in the biological function of OA chondrocytes. Our data found that miR-520c-3p was under-expressed in OA cartilage tissues and chondrocytes. Overexpression of miR-520c-3p could increase the proliferation and reduce the apoptosis and inflammation of OA chondrocytes, indicating that miR-520c-3p might be an effective strategy to alleviate OA progression.

A large number of studies have shown that miRNA can inhibit the progression of OA. For example, miR- 


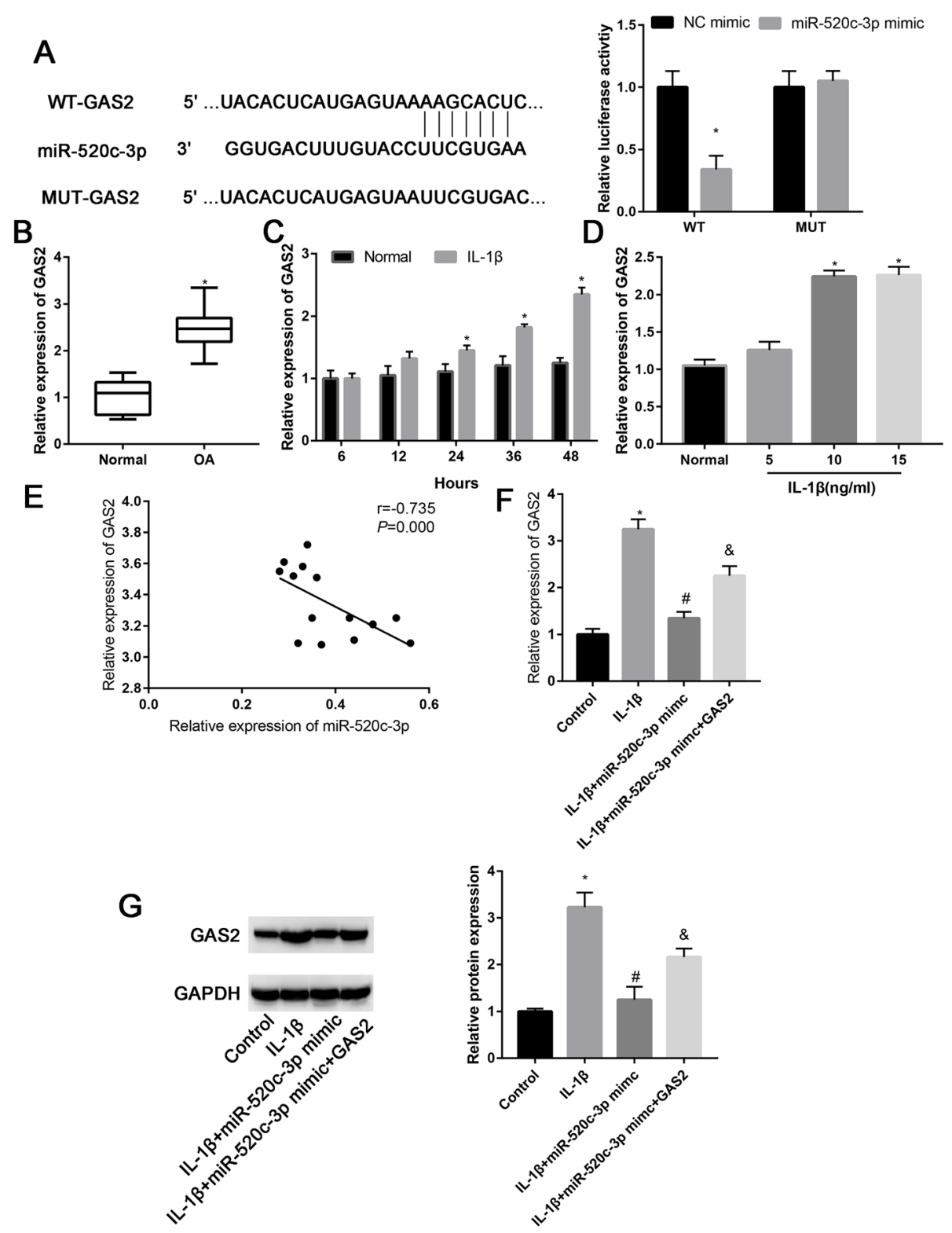

Fig. 4 GAS2 is a target gene of miR-520c-3p. a Putative binding sites between miR-520c-3p and GAS2. b The mRNA expression level of GAS2 in normal and OA cartilage. c Relative miR-520c-3p expression in normal and IL-1 $\beta$-stimulated chondrocytes at 6, 12, 24, 36, and 72 h. d Relative miR-520c-3p expression in different concentration IL-1 $\beta$-stimulated chondrocytes. e Correlation analysis between miR-520c-3p expression and GAS2 expression in normal and OA cartilage. qRT-PCR was applied to assess the mRNA level of GAS2 in control, IL-1 $\beta$, IL-1 1 + NC mimic, and IL$1 \beta+$ miR-520c-3p mimic groups. $\mathbf{f}$ Western blot assay and quantitative analysis the gray value of GAS2 expression in chondrocytes that treated with IL-1 $\beta, I L-1 \beta+N C$ mimic, and IL-1 $\beta+$ miR-520c-3p mimic

144-3p ameliorates the progression of OA through blocking the MAPK, PI3K/Akt, and NF-kB signaling pathways. Another study conducted by Cao et al. [18] found that decreased miR-214-3p activates NF- $\kappa B$ pathway and aggravates OA progression.

In this study, we found that miR-520c-3p was significantly downregulated in OA-affected cartilage and IL$1 \beta$-treated chondrocytes. The under-expressed miR- 520c-3p suggested that miR-520c-3p play a protective role in OA progression. miR-520c-3p implicated in many biological processes, including cholangiocarcinoma progression [15], lung injury [19], and inflammation response [20]. However, the role of miR-520c-3p for OA progression was unknown. We found that miR-520c-3p could inhibit the IL-1 $\beta$-stimulated chondrocytes' apoptosis, inflammation response, and ECM degradation. 


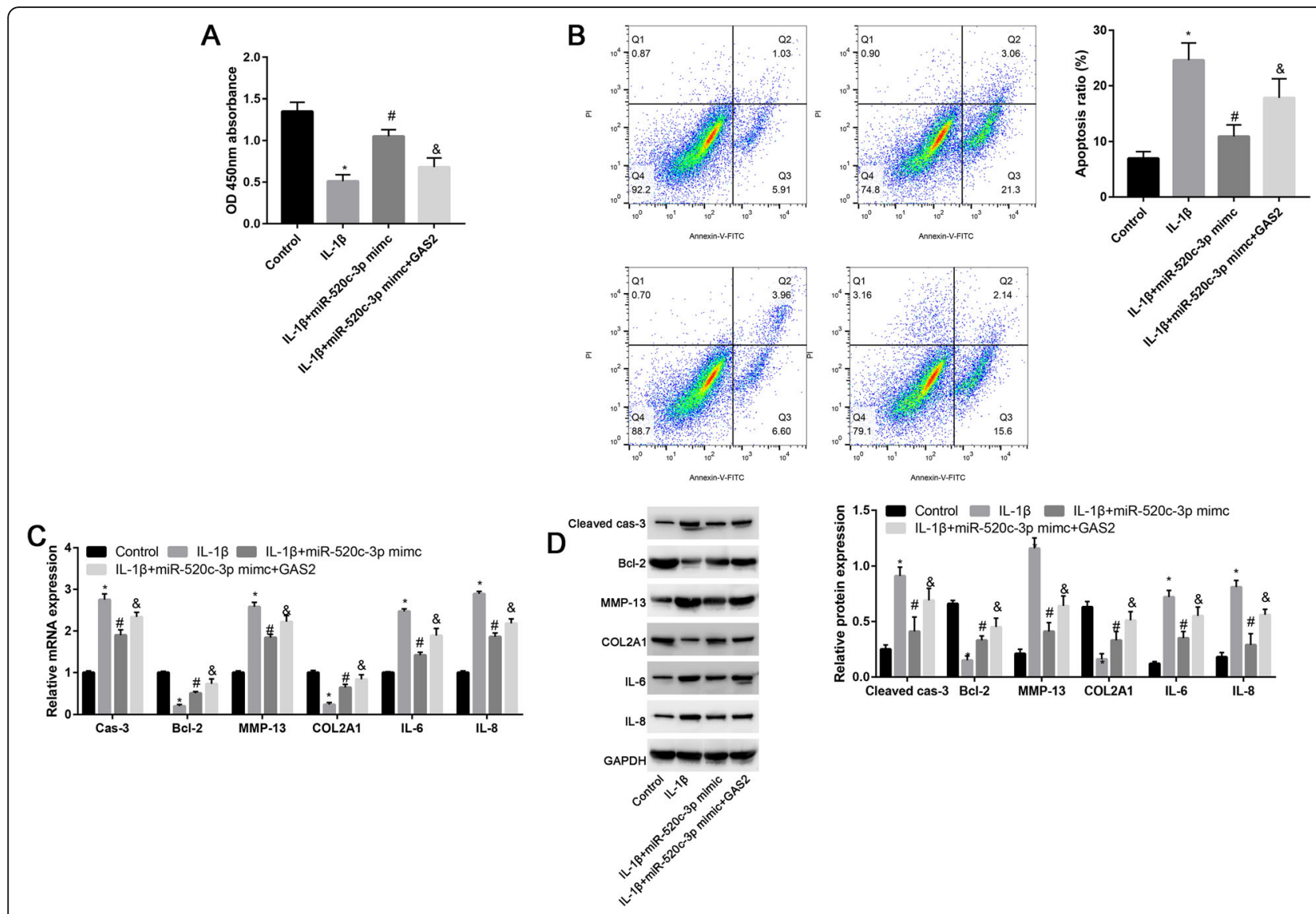

Fig. 5 Overexpression of GAS2 partially reverses the protective effects miR-520c-3p on IL-1 $\beta$-stimulated chondrocytes. a Cell viability in control, NC mimic, miR-520c-3p mimic, or miR-520c-3p mimic plus GAS2 overexpression plasmid. b Quantitative analysis of apoptosis rates in each group. c qRT-PCR was performed to assess the caspase-3, Bcl-2, MMP-13, COL2A1, IL-6, and IL-8 in control, IL-1 $\beta, I L-1 \beta+$ miR-520c-3p mimic, and IL-1 $\beta+$ miR-520c-3p mimic +GAS2 groups. $\mathbf{d}$ Western blot assay was performed to examine the protein expression levels (cleaved-caspase-3, Bcl-2, MMP-

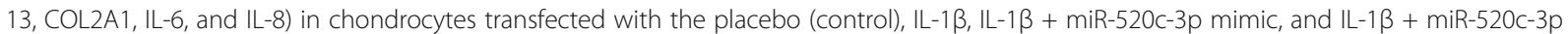
mimic + GAS2 groups

Additionally, our data showed that GAS2 was a target of miR-520c-3p. GAS2 was initially identified on account of its high level of expression in murine fibroblasts under growth arrest conditions. In the year of 1999, Lee et al. [17] found that GAS2 is a multifunctional gene involved in the regulation of apoptosis and chondrogenesis in the developing mouse limb. Recently, Kong et al. [21] found that GAS2 promotes cell proliferation and invasion and suppresses apoptosis in pediatric T-cell acute lymphoblastic leukemia and activates Wnt/ $\beta$-Catenin pathway. Zhu et al. [22] also found that GAS2 suppresses hepatocarcinogenesis by intervention of cell cycle and p53-dependent apoptosis. In this study, GAS2 was initially measured by RT-PCR and was upregulated in OA-affected cartilage and IL-1 $\beta$-treated chondrocytes. Moreover, overexpression of GAS2 could partially reverse the miR-520c-3p on OA progression, including chondrocytes' apoptosis, inflammation response, and ECM degradation. The lack of in vivo studies constitutes the main limitation of this study. The exact downstream components of the signaling pathway remain unknown.

\section{Conclusion}

Combined with all the results, we proposed that miR520c-3p inhibited OA progression through inhibiting the GAS2 expression. The pro-proliferation, antiapoptosis, and anti-inflammatory functions of miR-520c-3p on OA chondrocytes suggested that miR-520c-3p might be a beneficial method to alleviate OA progression, which had an important clinical significance.

\section{Abbreviations}

miRNAs: MicroRNAs; OA: Osteoarthritis; CCK8: Cell counting kit 8; WB: Western blot; RT-PCR: Real-time polymerase chain reaction; NSAI Ds: Nonsteroidal anti-inflammatory drugs; PRP: Platelet-rich plasma; HA: Hyaluronic acid; TNF-a: Tumor necrosis factor-a; IL-1 13: Interleukin 1 beta; GAS2: Growth arrest-specific 2; DMEM: Dulbecco's modified Eagle's medium; BCA: Bicinchoninic acid; PI: Propidium iodide; Annexin: V-fluorescein isothiocyanate; 3'UTR: $3^{\prime}$ untranslated region; WT: Wild-type; MUT: Mutant 


\section{Acknowledgements}

None.

\section{Authors' contributions}

Le Peng and Ming Deng conceived and designed the experiments; Yonggang $\mathrm{Ma}$ and Wei Hu performed the experiments and contributed reagents/materials/analysis tools; Le Peng and Fan Liang wrote the paper. All authors read and approved the final manuscript.

\section{Funding}

None.

\section{Availability of data and materials}

All data generated or analyzed during this study are included in this published article.

\section{Declarations}

Ethics approval and consent to participate

Our research was approved by the Ethic Committee of Renmin Hospital of Wuhan University.

\section{Consent for publication}

Informed consent was obtained from all patients.

\section{Competing interests}

The authors declare that they have no competing interests.

Received: 14 April 2021 Accepted: 6 May 2021

Published online: 29 May 2021

\section{References}

1. Glyn-Jones S, Palmer AJ, Agricola R, et al. Osteoarthritis. Lancet (London, England). 2015;386(9991):376-87.

2. Sacitharan PK. Ageing and osteoarthritis. Subcell Biochem. 2019;91:123-59. https://doi.org/10.1007/978-981-13-3681-2 6.

3. Nelson AE. Osteoarthritis year in review 2017: clinical. Osteoarthr Cartil. 2018; 26(3):319-25. https://doi.org/10.1016/j.joca.2017.11.014.

4. Bijlsma JW, Berenbaum F, Lafeber FP. Osteoarthritis: an update with relevance for clinical practice. Lancet (London, England). 2011;377(9783): 2115-26.

5. Xia B, Di C, Zhang J, Hu S, Jin H, Tong P. Osteoarthritis pathogenesis: a review of molecular mechanisms. Calcif Tissue Int. 2014;95(6):495-505. https://doi.org/10.1007/s00223-014-9917-9.

6. Geyer M, Schönfeld C. Novel insights into the pathogenesis of osteoarthritis. Curr Rheumat Rev. 2018;14(2):98-107. https://doi.org/10.2174/1573397113 666170807122312

7. Musumeci G, Castrogiovanni P, Trovato FM, Weinberg A, al-Wasiyah M, Alqahtani $M$, et al. Biomarkers of chondrocyte apoptosis and autophagy in osteoarthritis. Int J Mol Sci. 2015;16(9):20560-75. https://doi.org/10.3390/ ijms160920560.

8. Rim YA, Nam Y, Ju JH. The role of chondrocyte hypertrophy and senescence in osteoarthritis initiation and progression. Int J Mol Sci. 2020; 21(7):2358.

9. Guilak F, Nims RJ, Dicks A, Wu CL, Meulenbelt I. Osteoarthritis as a disease of the cartilage pericellular matrix. Matrix Biol. 2018;71-72:40-50.

10. Wu Y, Lu X, Shen B, Zeng $Y$. The therapeutic potential and role of miRNA, IncRNA, and circRNA in osteoarthritis. Curr Gene Ther. 2019:19(4):255-63. https://doi.org/10.2174/1566523219666190716092203.

11. Zhang W, Hu C, Zhang C, Luo C, Zhong B, Yu X. MiRNA-132 regulates the development of osteoarthritis in correlation with the modulation of PTEN/ PI3K/AKT signaling. BMC Geriatr. 2021;21(1):175. https://doi.org/10.1186/s12 877-021-02046-8.

12. Zhang $P$, Gao G. microRNA-130b downregulation potentiates chondrogenic differentiation of bone marrow mesenchymal stem cells by targeting SOX9. Braz J Med Biol Res. 2021;54(4):e10345.

13. Sun $L Z$, Jiang $Y Z$, Luan $S X$, Shi $Y L$, Wang $Q$. Long non-coding RNA FGD5AS1 promotes cell viability and metastasis in osteosarcoma by regulating miR-520c-3p. J Biol Regul Homeost Agents. 2021;35(1):323-9. https://doi. org/10.23812/20-644-L
14. Jiao Y, Zhao D, Gao F, Hu X, Hu X, Li M, et al. MicroRNA-520c-3p suppresses vascular endothelium dysfunction by targeting RELA and regulating the AKT and NF-KB signaling pathways. J Physiol Biochem. 2021;77(1):47-61. https://doi.org/10.1007/s13105-020-00779-5.

15. Li J, Jiang X, Li Z, Huang L, Ji D, Yu L, et al. SP1-induced HOXD-AS1 promotes malignant progression of cholangiocarcinoma by regulating miR520c-3p/MYCN. Aging. 2020;12(16):16304-25. https://doi.org/10.18632/a ging. 103660.

16. Ragni E, Coluccio A, Rolli E, Rodriguez-Peña JM, Colasante G, Arroyo J, et al. GAS2 and GAS4, a pair of developmentally regulated genes required for spore wall assembly in Saccharomyces cerevisiae. Eukaryot Cell. 2007;6(2): 302-16. https://doi.org/10.1128/EC.00321-06

17. Lee KK, Tang MK, Yew DT, et al. gas2 is a multifunctional gene involved in the regulation of apoptosis and chondrogenesis in the developing mouse limb. Dev Biol. 1999;207(1):14-25. https://doi.org/10.1006/dbio.1998.9086.

18. Cao $Y$, Tang $S$, Nie $X$, et al. Decreased miR-214-3p activates NF-kB pathway and aggravates osteoarthritis progression. EBioMedicine. 2021;65:103283. https://doi.org/10.1016/j.ebiom.2021.103283.

19. Li NN, Cao T, Yu F, Luo L, Liu P. MiR-520c-3p alleviates LPS-induced A549 cell and mice lung injury via targeting NLRC5. Pharmazie. 2020;75(6):275-8.

20. Liu Z, Zhao X, Shan H, Gao H, Wang P. microRNA-520c-3p suppresses NLRP3 inflammasome activation and inflammatory cascade in preeclampsia by downregulating NLRP3. Inflamm Res. 2019;68(8):643-54.

21. Kong Y, Zhao S, Tian H, Hai Y. GAS2 promotes cell proliferation and invasion and suppresses apoptosis in pediatric T-cell acute lymphoblastic leukemia and activates Wnt/ß-catenin pathway. Onco Targets Ther. 2020;13:1099-108. https://doi.org/10.2147/OTT.S236854.

22. Zhu RX, Cheng ASL, Chan HLY, Yang DY, Seto WK. Growth arrest-specific gene 2 suppresses hepatocarcinogenesis by intervention of cell cycle and p53-dependent apoptosis. World J Gastroenterol. 2019;25(32):4715-26. https://doi.org/10.3748/wjg.v25.i32.4715.

\section{Publisher's Note}

Springer Nature remains neutral with regard to jurisdictional claims in published maps and institutional affiliations.
Ready to submit your research? Choose BMC and benefit from:

- fast, convenient online submission

- thorough peer review by experienced researchers in your field

- rapid publication on acceptance

- support for research data, including large and complex data types

- gold Open Access which fosters wider collaboration and increased citations

- maximum visibility for your research: over $100 \mathrm{M}$ website views per year

At BMC, research is always in progress.

Learn more biomedcentral.com/submission 